\title{
Numerical Analysis of the Burger Equation using Finite Differences
} Saad A. Manna

Badran J. Salem

College of Computer Science and Mathematics College of Basic Education

University of Mosul, Iraq

\section{Received on: 23/07/2006}

Accepted on: 04/10/2006

\section{ABSTRACT}

The Burger equation had been solved numerically by using two finite differences methods. The first is explicit scheme method and the second is Crank-Nicholson method. A comparison had been made between the two and we find that the Crank-Nicholson method is the best and most accurate than the explicit scheme method, as it is shown in (Table 1). Also the numerical stability for both methods has been made, the explicit scheme method is conditionally stable and the condition is $\alpha \leq \frac{1}{2 \varepsilon}$, while CrankNicholson method is unconditionally stable.

Keywords: Numerical Analysis, Finite Differences, explicit scheme method, Crank-Nicholson method, Burger Equation.

$$
\begin{aligned}
& \text { التحليل العدادي لمعادلة Burger باستخدام الفروقات المنتهية } \\
& \text { بران جاسم سالم } \\
& \text { سعد عبد الله مناع } \\
& \text { كلية التربية الأساسية، جامعة الدوصل } \\
& \text { كلية علوم الحاسبات والرياضيات، جامعة الدوصل } \\
& \text { تاريخ قبول البحث: 2006/10/4 } \\
& \text { تاريخ استلام البحث: 2006/7/23 }
\end{aligned}
$$

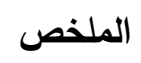

لقد تم حل معادلة Burger باستخدام نوعين من طرائق الفرو قات المنتهية،الأولى هي

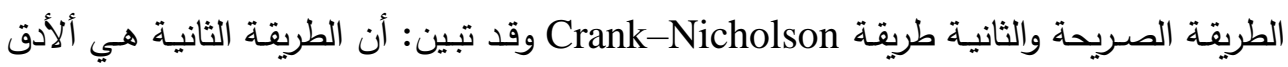

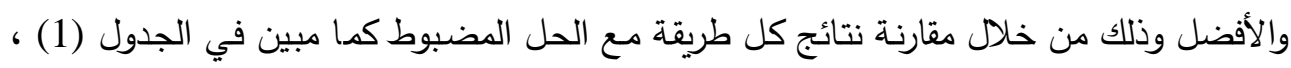

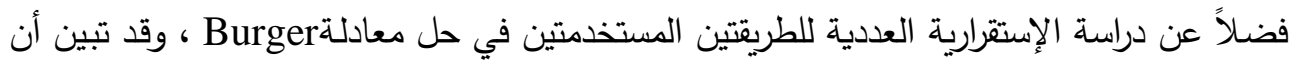

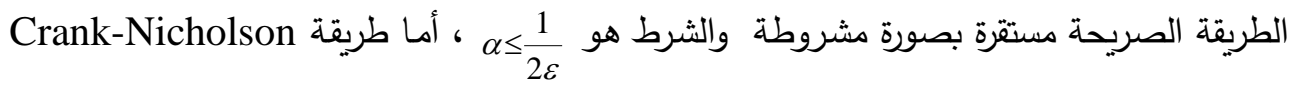
فهي مستقرة من دون شروط. Crank- الكلمات المفتاحية: التحليل العددي، طرائق الفروقات المنتهية، الطريقة الصريحة، طريقة 


\section{Introduction}

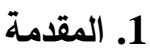

تعتبر المعادلات التفاضلية خير وسيلة لوصف معظم المسائل الهندسية والرياضية والعلمية على حد سواء، إذ يتضح ذلك جليا في وصف عمليات انتقال الحرارة، جريان الموائع، الحركة

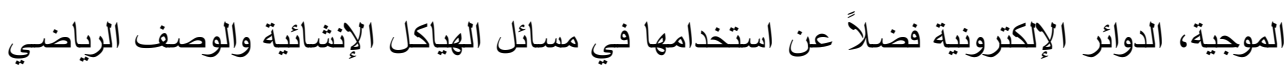

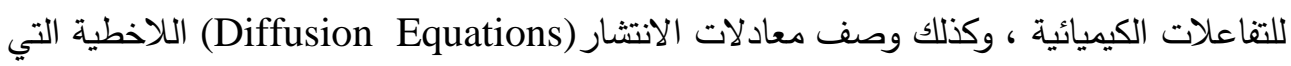
تؤدي دورا مهما في الأنظمة الحركية المشتتة ومن الأمثلة على معادلات الانتثار اللاخطية معادلة Burger

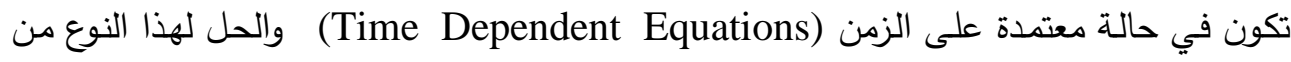
المسائل يكون في منطقة مفتوحة R التي تخضع لشروط حدودية ، هذه المسائل تتنج في دراسة

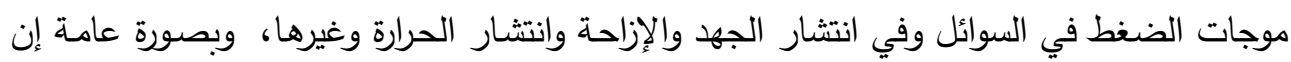
إمكانية الحصول على حلول تحليلية Analytical Solution) لمسائل الانتثار هي معقدة كثيرا

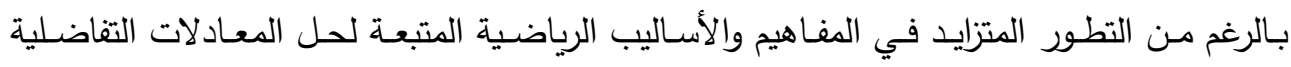

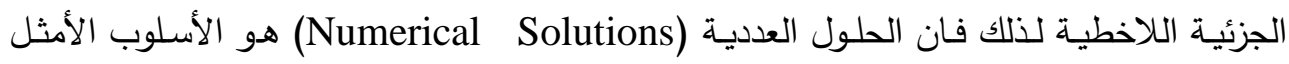
لاراسة خواص هذا النوع من المعادلات [7]. أن دراسة الخواص العامة لمعادلة Burger ضرورية لمعادلات مكانيك الموائع المشتركة. التي تبين التركيب ووجه التقارب كما هو الحال في معادلات( نافر - ستوكس - Navier ) .[5] stokes

في عام (1990) درس Kakuda \&Tosaka [5 تقريب العناصر الحدودية العامة

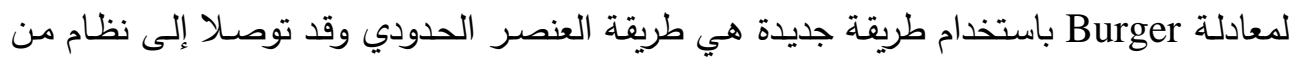
المعادلات شبه خطية تم حلها ضمنيا باستخدام طرائق تكرارية بسيطة. وفي عام (1994) درس Estevez [4] التتاظر غير التقليدي (غير القياسي) وطريقة singular manifold لمعادلة Burger إذ استخدم طرائق مباشرة لإيجاد التخفيضات المشابهة

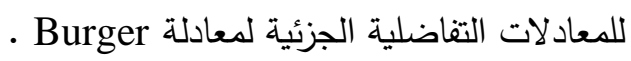

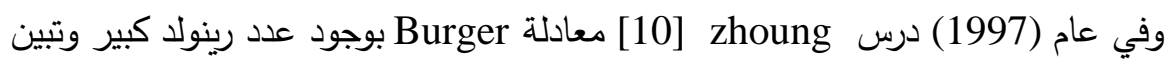

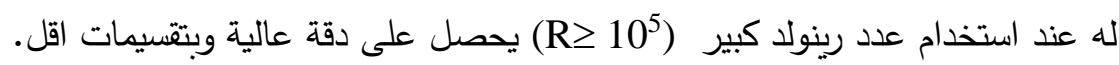




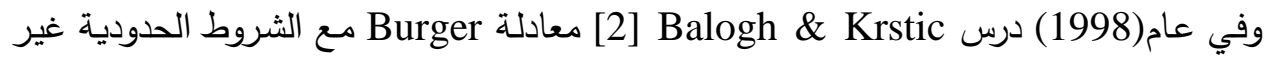
الخطية وبين ان المعادلة تضمن استقرارية محاذية عامة واستقرارية آسية شبه عامة في

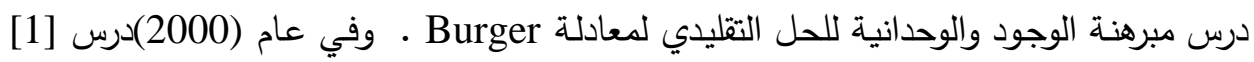
Atoull \& King

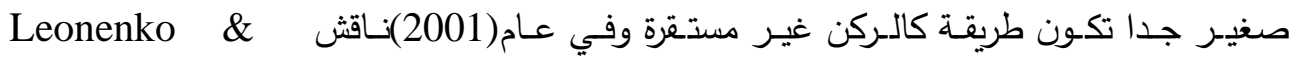
كeluikovn كذلك في عام (2001) درس Balogh \& Gilliam \& Shubov [3 الحلول الساكنة لمعادلة Burger

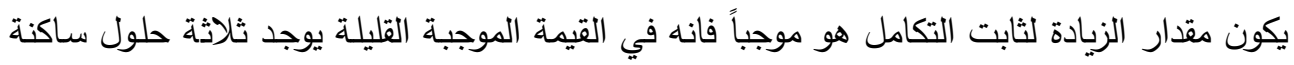

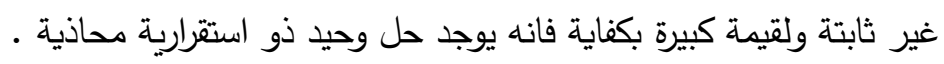

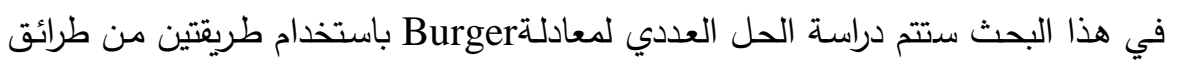
الفروقات المنتهية (Finite Differences Methods) ، الأولى الطريقة الصريحة ( Explicit

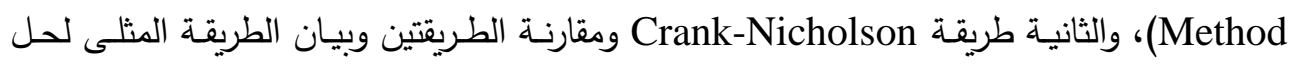
معادلة Burger ، كذلك دراسة الاستقرارية العددية لكلتا الطريقتين.

Mathematical Model

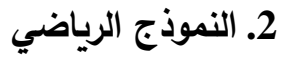
أن من معادلات الانتشار المعروفة معادلة Burger غير الخطية آلاتية:[9]

$$
\frac{\partial \mathrm{u}}{\partial \mathrm{t}}+\mathrm{u} \frac{\partial \mathrm{u}}{\partial \mathrm{x}}-\varepsilon \frac{\partial^{2} \mathrm{u}}{\partial \mathrm{x}^{2}}=0
$$

$$
\mathrm{u}(\mathrm{x}, 0)=\mathrm{u}_{0}(\mathrm{x})
$$$$
\mathrm{u}(1, \mathrm{t})=\mathrm{g}(\mathrm{t})
$$$$
\mathrm{u}(0, \mathrm{t})=\mathrm{f}(\mathrm{t})
$$$$
0<\mathrm{x}<1 \quad \mathrm{t}>0 \quad \varepsilon>0
$$

المعادلة(1) تصف المجال لامتداد الموجة أو الذبذبة في أنظمة مبددة تلعب دورا مهما في 
ان من اكثر الطرائق استخدماً لحل المعادلات التفاضلية الجزئية هي طريقة الفروقات المنتهية (Finite Differences) التي تعطي تقريبات جيدة للحل.

\section{Explicit Method}

3.الطريقة الصريحة

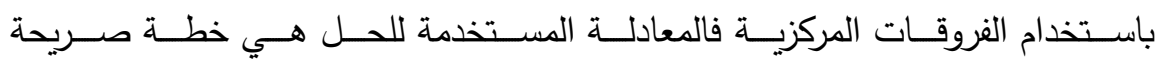

والتي عن طريقها يتم ايجاد قيمـة الدالة عند نقاط المشبك

أي قيمة الدالة غير المعروفة (m , n+1)

النقاط

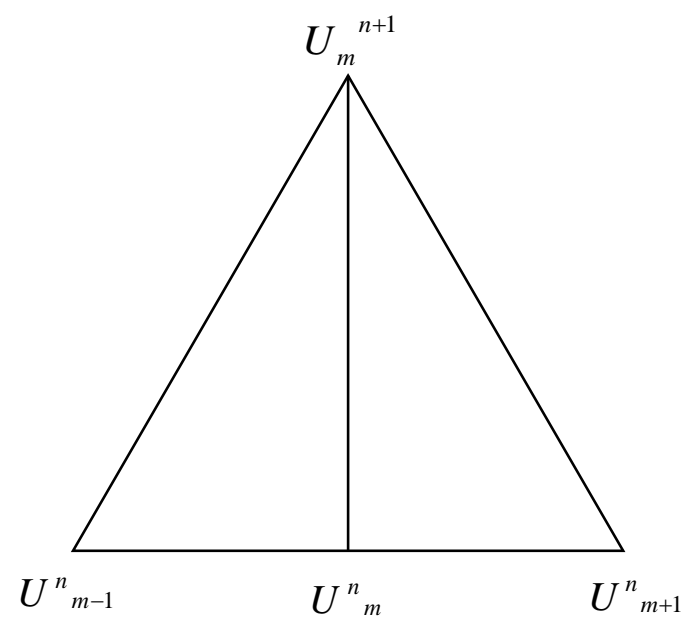

(1) (1) (1)

حيث نقسم المستوي إلى شبكة من المستطيلات ذات جوانب h,k نقاط تقاطع هذه

الخطوط تعرف بنقاط الثبكة(Grid points) والأسلوب العام لحل المعادلة التفاضلية الجزئية بهذه الطريقة هو الحصول على الحل عند نقاط الشبكة ، ونقاط الشبكة هذه قد تكون ناتجة عن تقاطع الخطوط المستطيلة أو المربعة بحيث: 


$$
\begin{aligned}
& x=m h \\
& t=n k \\
& m=0,1,2,3 \ldots \ldots \ldots \ldots \frac{1}{h} \\
& n=0,1,2,3 \ldots \ldots \ldots \ldots . . \frac{1}{k} .
\end{aligned}
$$

وعليه يكون التقريب العددي للمشتقة الجزئية الأولى والثانية للدالة (u) نسبة إلى (x) و (t) وباستخدام مفكوك تايلر كما يأتي][7]:

$$
\frac{\partial \mathrm{u}}{\partial \mathrm{x}}=\frac{\mathrm{U}_{\mathrm{m}+1}^{\mathrm{n}}-\mathrm{U}_{\mathrm{m}-1}^{\mathrm{n}}}{2 \mathrm{~h}}
$$

$\frac{\partial^{2} \mathrm{u}}{\partial \mathrm{x}^{2}}=\frac{\mathrm{U}_{\mathrm{m}+1}^{\mathrm{n}}-2 \mathrm{U}_{\mathrm{m}}^{\mathrm{n}}+\mathrm{U}_{\mathrm{m}-1}^{\mathrm{n}}}{\mathrm{h}^{2}}$

$\frac{\partial \mathrm{u}}{\partial \mathrm{t}}=\frac{\mathrm{U}_{\mathrm{m}}^{\mathrm{n}+1}-\mathrm{U}_{\mathrm{m}}^{\mathrm{n}}}{\mathrm{k}}$

$$
\frac{\partial^{2} \mathrm{u}}{\partial \mathrm{t}^{2}}=\frac{\mathrm{U}_{\mathrm{m}}^{\mathrm{n}+1}-2 \mathrm{U}_{\mathrm{m}}^{\mathrm{n}}+\mathrm{U}_{\mathrm{m}}^{\mathrm{n}-1}}{\mathrm{k}^{2}}
$$

الآن نأخذ معادلة( Burger )المتمثلة بالمعادلة (1) وباستخدام الفرو قات المركزية (4)

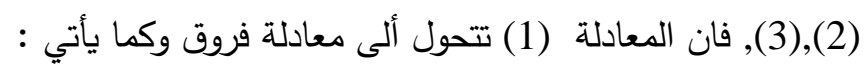

$$
\begin{aligned}
& \frac{1}{k}\left(U_{m}^{n+1}-U_{m}^{n}\right)+U_{m}^{n}\left[\frac{1}{2 h}\left(U_{m+1}^{n}-U_{m-1}^{n}\right)\right]=\frac{\varepsilon}{h^{2}}\left(U_{m+1}^{n}-2 U_{m}^{n}+U_{m-1}^{n}\right) \ldots . .(6) \\
& \text { وبضرب المعادلة (6) بـ نحصل على: } \\
& U_{m}^{n+1}-U_{m}^{n}+\frac{k}{2 h} U_{m}^{n}\left(U_{m+1}^{n}-U_{m-1}^{n}\right)=\varepsilon \alpha U_{m+1}^{n}-2 \varepsilon \alpha U_{m}^{n}+\varepsilon \alpha U_{m-1}^{n} \\
& \alpha=\frac{\mathrm{k}}{\mathrm{h}^{2}} \stackrel{ }{\mathrm{N}}
\end{aligned}
$$


$U_{m}^{n+1}=U_{m}^{n}-\frac{k}{2 h} U_{m}^{n}\left(U_{m+1}^{n}-U_{m-1}^{n}\right)+\varepsilon \alpha U_{m+1}^{n}-2 \varepsilon \alpha U_{m}^{n}+\varepsilon \alpha U_{m-1}^{n}$

$\Rightarrow U_{m}^{n+1}=(1-2 \varepsilon \alpha) U_{m}^{n}-\frac{k}{2 h} U_{m}^{n}\left(U_{m+1}^{n}-U_{m-1}^{n}\right)+\varepsilon \alpha\left(U_{m+1}^{n}+U_{m-1}^{n}\right) \ldots .(7)$

حيث أن المعادلة(7) هي التي سوف تستخدم للحل بالطريقة الصريحة.

\section{Crank Nicholson يقة}

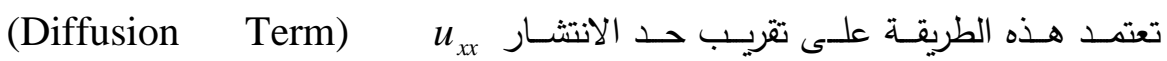

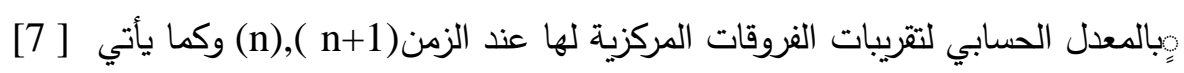

$\frac{\partial \mathrm{u}}{\partial \mathrm{t}}=\frac{\mathrm{U}_{\mathrm{m}}^{\mathrm{n}+1}-\mathrm{U}_{\mathrm{m}}^{\mathrm{n}}}{\mathrm{k}}$

$\frac{\partial^{2} \mathrm{u}}{\partial \mathrm{x}^{2}}=\frac{1}{2}\left[\frac{\mathrm{U}_{\mathrm{m}+1}^{\mathrm{n}+1}-2 \mathrm{U}_{\mathrm{m}}^{\mathrm{n}+1}+\mathrm{U}_{\mathrm{m}-1}^{\mathrm{n}+1}}{\mathrm{~h}^{2}}+\frac{\mathrm{U}_{\mathrm{m}+1}^{\mathrm{n}}-2 \mathrm{U}_{\mathrm{m}}^{\mathrm{n}}+\mathrm{U}_{\mathrm{m}-1}^{\mathrm{n}}}{\mathrm{h}^{2}}\right]$

ألان نأخذ معادلة Burger المتمثلة بالمعادلة (1) وباستخدام المعادلتين (8) و (9) نحصل على: $\frac{1}{k}\left(U_{m}^{n+1}-U_{m}^{n}\right)=\frac{\varepsilon}{2 h^{2}} \cdot\left[\left(U_{m+1}^{n+1}-2 U_{m}^{n+1}+U_{m-1}^{n+1}\right)+\left(U_{m+1}^{n}-2 U_{m}^{n}+U_{m-1}^{n}\right)\right]$

$$
-U_{m}^{n}\left[\frac{1}{2 h}\left(U_{m+1}^{n}-U_{m-1}^{n}\right)\right]
$$

$$
\begin{aligned}
\left(U_{m}^{n+1}-U_{m}^{n}\right) & =\frac{k \varepsilon}{2 h^{2}} \cdot\left[\left(U_{m+1}^{n+1}-2 U_{m}^{n+1}+U_{m-1}^{n+1}\right)+\left(U_{m+1}^{n}-2 U_{m}^{n}+U_{m-1}^{n}\right)\right] \\
& -U_{m}^{n}\left[\frac{k}{2 h}\left(U_{m+1}^{n}-U_{m-1}^{n}\right)\right]
\end{aligned}
$$

$$
\alpha=\frac{k}{h^{2}}
$$




$$
\begin{aligned}
& U_{m}^{n+1}-U_{m}^{n}=\frac{\alpha \varepsilon}{2} U_{m+1}^{n+1}-\alpha \varepsilon U_{m}^{n+1}+\frac{\alpha \varepsilon}{2} U_{m-1}^{n+1}+\frac{\alpha \varepsilon}{2} U_{m+1}^{n}-\alpha \varepsilon U_{m}^{n}+\frac{\alpha \varepsilon}{2} U_{m-1}^{n} \\
& \quad-\frac{\alpha h}{2} U_{m}^{n} \cdot U_{m+1}^{n}+\frac{\alpha h}{2} U_{m}^{n} U_{m-1}^{n} \\
& \Rightarrow(1+\alpha \varepsilon) U_{m}^{n+1}-\frac{\alpha \varepsilon}{2}\left(U_{m+1}^{n+1}+U_{m-1}^{n+1}\right)=\frac{\alpha \varepsilon}{2}\left(U_{m+1}^{n}+U_{m-1}^{n}\right) \\
& +\left(1-\alpha \varepsilon-\frac{\alpha h}{2} U_{m+1}^{n}+\frac{\alpha h}{2} U_{m-1}^{n}\right) U_{m}^{n} \\
& \left.\left(\frac{2}{\alpha \varepsilon}+2\right) U_{m}^{n+1}-\left(U_{m+1}^{n+1}+U_{m-1}^{n+1}\right)=U_{m+1}^{n}+U_{m-1}^{n}\right) \\
& +\left(\frac{2}{\alpha \varepsilon}-2-\frac{h}{\varepsilon} U_{m+1}^{n}+\frac{h}{\varepsilon} U_{m-1}^{n}\right) U_{m}^{n}
\end{aligned}
$$

نلاحظ من المعادلة (14 ) أن الطرف الأيسر يتضمن ثلاث قيم غير معلومة للدلة (u)

عند الخطوة الزمنية الجديدة (n+1) بينما القيم في الطرف الأيمن كلها معلومة عند الخطوة الزمنية السابقة (n) لذلك فان ألمعادلة هي علاقة ضمنية . لزئه

مثال:

في حل المثال التالي نأخذ معادلة Burger المتمثلة بالمعادلة (1) بحيث: [6]

$u(x, t)=\operatorname{Sin} \pi x$

$$
u(1, t)=0
$$$$
u(0, t)=0
$$$$
0<x<1
$$$$
\varepsilon=0.1
$$$$
k=0.05
$$$$
h=0.1
$$ 


\begin{tabular}{|c|c|c|}
\hline EXPLICIT & Crank-Nicholson & Exact \\
$\boldsymbol{E}=0.1$ & $\boldsymbol{E}=0.1$ & $\varepsilon=0.1$ \\
$k=0.05$ & $k=0.05$ & $k=0.05$ \\
$h=0.1$ & $h=0.1$ & $h=0.1$ \\
\hline $\mathbf{0 . 0}$ & $\mathbf{0 . 0}$ & $\mathbf{0 . 0}$ \\
\hline $\mathbf{0 . 2 8 2 5}$ & $\mathbf{0 . 2 5 0 0}$ & $\mathbf{0 . 2 5 8 6}$ \\
\hline $\mathbf{0 . 4 6 6 6}$ & $\mathbf{0 . 5 0 8 9}$ & $\mathbf{0 . 5 0 0 0}$ \\
\hline $\mathbf{0 . 6 1 5 4}$ & $\mathbf{0 . 7 0 9 9}$ & $\mathbf{0 . 7 0 6 4}$ \\
\hline $\mathbf{0 . 7 1 4 5}$ & $\mathbf{0 . 8 6 0 1}$ & $\mathbf{0 . 8 5 9 9}$ \\
\hline $\mathbf{0 . 7 5 0 0}$ & $\mathbf{0 . 9 4 8 2}$ & $\mathbf{0 . 9 4 2 3}$ \\
\hline $\mathbf{0 . 7 1 2 1}$ & $\mathbf{0 . 9 2 9 9}$ & $\mathbf{0 . 9 3 7 4}$ \\
\hline $\mathbf{0 . 5 9 8 1}$ & $\mathbf{0 . 8 2 9 6}$ & $\mathbf{0 . 8 3 3 4}$ \\
\hline $\mathbf{0 . 4 1 5 1}$ & $\mathbf{0 . 6 1 9 7}$ & $\mathbf{0 . 6 2 8 9}$ \\
\hline $\mathbf{0 . 1 8 1 0}$ & $\mathbf{0 . 3 4 1 1}$ & $\mathbf{0 . 3 3 9 3}$ \\
\hline $\mathbf{0 . 0}$ & $\mathbf{0 . 0}$ & $\mathbf{0 . 0}$ \\
\hline
\end{tabular}

(1) الجدول

يوضح مقارنة الحل العددي للطريقة الصريحة وطريقة Crank-Nicholson مع الحل الحقيقي.

\begin{tabular}{|c|c|c|}
\hline $\begin{array}{c}\boldsymbol{E}=0.01 \\
k=0.05\end{array}$ & $\begin{array}{c}\boldsymbol{E}=0.001 \\
k=0.05 \\
h=0.1\end{array}$ & $\begin{array}{c}\boldsymbol{E}=0.0001 \\
k=0.1\end{array}$ \\
\hline $\mathbf{0 . 0}$ & $\mathbf{0 . 0}$ & $h=0.1$ \\
\hline $\mathbf{0 . 2 8 8 6 6 8 6 7 4}$ & $\mathbf{0 . 2 9 0 4 5 6 8 4 3}$ & $\mathbf{0 . 2 9 9 8 5 2 5 7 6}$ \\
\hline $\mathbf{0 . 4 8 8 3 2 8 6 5 5}$ & $\mathbf{0 . 5 2 9 4 9 5 3 9 5}$ & $\mathbf{0 . 5 6 9 1 0 4 5 5 3}$ \\
\hline $\mathbf{0 . 8 0 5 0 5 7 3 8 2}$ & $\mathbf{0 . 8 6 3 6 1 5 2 6 4}$ & $\mathbf{0 . 8 9 1 2 4 8 6 3 4}$ \\
\hline $\mathbf{0 . 9 0 6 9 0 1 3 2 0}$ & $\mathbf{0 . 9 6 2 4 8 6 1 5 8}$ & $\mathbf{0 . 9 7 1 5 9 7 6 0 5}$ \\
\hline $\mathbf{0 . 9 5 2 4 9 3 7 9 5}$ & $\mathbf{0 . 9 8 5 2 4 6 3 1 5}$ & $\mathbf{0 . 9 8 9 9 5 2 4 9 5}$ \\
\hline $\mathbf{0 . 9 0 1 8 1 0 6 2 1}$ & $\mathbf{0 . 9 5 8 6 1 4 7 2 3}$ & $\mathbf{0 . 9 6 9 2 4 5 8 6 3}$ \\
\hline $\mathbf{0 . 8 0 1 5 8 8 4 5 5}$ & $\mathbf{0 . 8 5 5 4 3 9 0 1 5}$ & $\mathbf{0 . 8 8 5 2 4 6 7 2 1}$ \\
\hline $\mathbf{0 . 4 8 1 2 3 7 1 5 0}$ & $\mathbf{0 . 5 1 5 8 9 4 2 8 3}$ & $\mathbf{0 . 5 5 8 3 1 4 9 7 2}$ \\
\hline $\mathbf{0 . 2 0 8 5 2 1 0 5 3}$ & $\mathbf{0 . 2 8 9 5 6 8 4 2 4}$ & $\mathbf{0 . 3 0 0 4 1 5 4 1 9}$ \\
\hline $\mathbf{0 . 0}$ & $\mathbf{0 . 0}$ & $\mathbf{0 . 0}$ \\
\hline
\end{tabular}

(2) (الجدول

يوضح الطريقة الصريحة بأخذ قيم مختلفة لـ ع (2010 0.0001, 0.001, 0.01 ) 


\section{4. تحليل الأستقرارية في الطريقة الصريحة لمعادلة Burger}

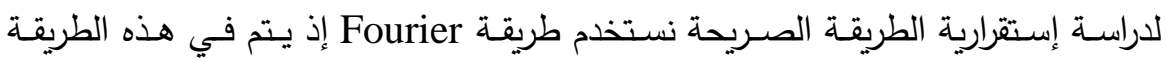

استبدال الحل

نعوض عن $\psi$

$\frac{1}{k}\left(\psi(t+k) e^{j \beta x}-\psi(t) e^{j \beta x}\right)+\psi(t) e^{j \beta x} \frac{1}{2 h}\left[\psi(t) e^{j \beta(x+h)}-\psi(t)\right.$

$\left.\left.e^{j \beta(x-h)}\right)\right]=\frac{\varepsilon}{h^{2}}\left(\psi(t) e^{j \beta(x+h)}-2 \psi(t) e^{j \beta x}+\psi(t) e^{j \beta(x-h)}\right)$

$\Rightarrow \frac{1}{k}(\psi(t+k)-\psi(t)) e^{j \beta x}+\left[\frac{1}{2 h} \psi(t) \psi(t) e^{j \beta x} e^{j \beta x}\left(e^{j \beta h}\right.\right.$

$\left.\left.-e^{-j \beta h}\right)\right]=\frac{\varepsilon}{h^{2}} e^{j \beta x} \psi(t)\left(e^{j \beta h}-2+e^{-j \beta h}\right)$

بعد ضرب المعادلة السابقة بـ

و بغرض $\alpha=\frac{k}{h^{2}}$

$\psi(t+k)-\psi(t)+\frac{k}{2 h} \psi(t) \psi(t) e^{j \beta x}\left(e^{j \beta h}-e^{-j \beta h}\right)=\alpha \varepsilon \psi(t)\left(e^{j \beta h}-2+e^{-j \beta h}\right)$

$\Rightarrow \psi(\mathrm{t}+k)-\psi(t)=-\frac{k}{2 h} \psi(t) \psi(t) e^{j \beta h}(\cos (\beta h)+j \sin (\beta h))-(\cos (\beta h)-j \sin (\beta h))$

$+\alpha \varepsilon \psi(t)[(\cos (\beta h)+j \sin (\beta h))-2+(\cos (\beta h)-j \sin (\beta h))]$

$\Rightarrow \psi(t+k)-\psi(t)=-\frac{k}{2 h} \psi(t) \psi(t) e^{j \beta x}(2 j \sin (\beta h))$

$+\alpha \varepsilon \psi(t)(2 \cos (\beta h)-2)$

وبما أن الجزء الحقيقي هو الذي يؤدي الى الاستقرارية لذلك نهمل الجزء الخيالي فنحصل على: (t) (t)

$\psi(t)(1-\cos (\beta h))-2 \alpha \varepsilon=\psi(t) \psi(t+k)-$

$\Rightarrow \psi(t+k)=\psi(t)-2 \alpha \varepsilon \psi(t)\left(1-\left(1-2 \sin ^{2}\left(\frac{\beta h}{2}\right)\right)\right.$ 
$\Rightarrow \psi(t+k)=\psi(t)-2 \alpha \varepsilon \psi(t)\left(2 \sin ^{2}\left(\frac{\beta h}{2}\right)\right)$

$\Rightarrow \psi(t+k)=\psi(t)\left[1-4 \alpha \varepsilon\left(\sin ^{2}\left(\frac{\beta h}{2}\right)\right]\right.$

$\Rightarrow \psi(t+k) / \psi(t)=(1-4 \phi)=\zeta$

$\phi=\alpha \varepsilon \sin ^{2}\left(\frac{\beta h}{2}\right) \quad$ وبفرض

$\Rightarrow|\psi(\mathrm{t}+\mathrm{k}) / \psi(\mathrm{t})|=|\zeta|$

وبأخذ القيمة المطلقة للطرفين

حيث أنه كلما تقدم الحل من سطح مستوِ خاص لـ (t) $\psi$ فان | يجب أن لاتقترب من اللانهاية عندما t كبيرة وذلك للحصول على الاستقرارية [8]. من المعادلة (15) لنقيد (t)

$\Rightarrow 1 \leq|\psi(\mathrm{t}+\mathrm{k}) / \psi(\mathrm{t})|$

$|\zeta| \leq 1 \Rightarrow$

$\Rightarrow|1-4 \phi| \leq 1$

$\Rightarrow-1 \leq(1-4 \phi) \leq 1$

$(1-4 \phi) \leq 1$

في المتباينة المذكورة أنفاً نأخذ الطرف الأيمن منها

بالتعويض عن قيمة

$\left(1-4 \alpha \varepsilon\left(\sin ^{2}\left(\frac{\beta h}{2}\right)\right) \leq 1\right.$

تكون $\alpha \geq 0$ وهذٍ تكون دائما صحيحة . ولهذا ولكي تكون المعادلة (16) صحيحة نحتاج إلى اخذ الطرف الأيسر

$\Rightarrow-1 \leq\left(1-4 \alpha \varepsilon\left(\sin ^{2}\left(\frac{\beta h}{2}\right)\right)\right.$

$\Rightarrow-2 \leq-4 \alpha \varepsilon\left(\sin ^{2}\left(\frac{\beta h}{2}\right)\right)$ 
$\Rightarrow 2 \geq 4 \alpha \varepsilon\left(\sin ^{2}\left(\frac{\beta h}{2}\right)\right)$

لبعض قيم

$4 \varepsilon \alpha \leq 2$

$\alpha \leq \frac{1}{2 \varepsilon}$

إذا طريقة الفروقات المركزية لمعادلة بيركر تكون مستقرة عندما

Burger (2-1-3) تحليل الإستقرارية في طريقة Crank-Nicholson لمعادلة

نعوض عن

$\frac{1}{k}\left(\psi(t+k) e^{j \beta x}-\psi(t) e^{j \beta x}\right)=\frac{\varepsilon}{2 h^{2}}\left[\left(\psi(t+k) e^{j \beta(x+h)}-2 \psi(t+k) e^{j \beta x}\right.\right.$

$\left.\left.+\psi(t+k) e^{j \beta(x-h)}\right)+\left(\psi(t) e^{j \beta(x+h)}-2 \psi(t) e^{j \beta x}+\psi(t) e^{j \beta(x-h)}\right)\right]$

$-\frac{1}{2 h} \psi(t) e^{j \beta x}\left(\psi(t) e^{j \beta(x+h)}-\psi(t) e^{j \beta(x-h)}\right)$

$$
\begin{array}{r}
k \text { وبضرب طرفي المعادلة بفرض } \quad \text { وب } \\
\alpha=\frac{k}{h^{2}}
\end{array}
$$

$(\psi(t+k)-\psi(t)) e^{j \beta x}=\frac{\varepsilon \alpha}{2} e^{j \beta x}\left(\left(\psi(t+k) e^{j \beta h}-2 \psi(t+k)+\psi(t+k)\right.\right.$

$\left.e^{-j \beta h}\right)+\frac{\varepsilon \alpha}{2} \psi(t) e^{j \beta x}\left(e^{j \beta h}-2+e^{-j \beta h}\right)-\frac{k}{2 h} \psi(t) e^{j \beta x}$

$\psi(t) e^{j \beta x}\left(e^{j \beta h}-e^{-j \beta h}\right)$

ألان نضرب في 


$$
\begin{aligned}
& \begin{aligned}
\psi(t+k)-\psi(t)= & \frac{\varepsilon \alpha}{2} \psi(t+k)\left(e^{j \beta h}-2+e^{-j \beta h}\right)+\frac{\varepsilon \alpha}{2} \psi(t)\left(e^{j \beta h}-2+\right. \\
\left.e^{-j \beta h}\right) & \\
& -\frac{k}{2 h} \psi(t) \psi(t) e^{j \beta x}\left(e^{j \beta h}-e^{-j \beta h}\right)
\end{aligned}
\end{aligned}
$$$$
\psi(\mathrm{t}+k)-\psi(t)=\frac{\varepsilon \alpha}{2} \psi(t+k)[(\cos (\beta h)+j \sin (\beta h))-2+(\cos (\beta h)-j \sin (\beta h))]
$$$$
+\frac{\varepsilon \alpha}{2} \psi(t)[(\cos (\beta h)+j \sin (\beta h))-2+(\cos (\beta h)-j \sin (\beta h))]
$$$$
-\frac{\mathrm{k}}{2 \mathrm{~h}} \psi(\mathrm{t}) \mathrm{e}^{\mathrm{j} \beta \mathrm{x}} \psi(\mathrm{t})[(\cos (\beta h)+j \sin (\beta h))-(\cos (\beta h)-j \sin (\beta h))]
$$$$
\Rightarrow \psi(t+k))-\psi(t)=\frac{\varepsilon \alpha}{2} \psi(t+k)[(2 \cos (\beta h)-2)]
$$$$
+\frac{\varepsilon \alpha}{2} \psi(t)[(2 \cos (\beta h)-2)]-\frac{k}{2 h} \psi(t) e^{j \beta x} \psi(t)[2 j \sin (\beta h)]
$$

وبما أن الجزء الحقيقي هو الذي يؤدي الى الاستقرارية لذلك نهمل الجزء الخيالي فنحصل على:

$$
\psi(t+k)-\psi(t)=-\varepsilon \alpha(1-\cos (\beta h))[\psi(t+k)+\psi(t)]
$$

$\Rightarrow \psi(t+k)-\psi(t)=-\varepsilon \alpha\left[1-\left(1-2 \sin ^{2}\left(\frac{\beta h}{2}\right)\right)\right][\psi(t+k)+\psi(t)]$

$\Rightarrow \psi(t+k)-\psi(t)=-2 \alpha \varepsilon\left(\sin ^{2}\left(\frac{\beta h}{2}\right)\right)[\psi(t+k)+\psi(t)]$

$$
\phi=-2 \alpha \varepsilon \sin ^{2}\left(\frac{\beta h}{2}\right) \text { نفرض }
$$

$\Rightarrow \psi(t+k)=\psi(t)+\phi[\psi(t+k)+\psi(t)]$ 
بالقسمة على (t)

$\Rightarrow \psi(t+k) / \psi(t)=1+[\phi(\psi(t+k)+\psi(t))] / \psi(t)$

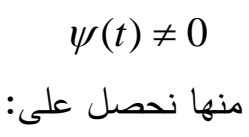

$\psi(t+k) / \psi(t)=1+\phi \quad(\psi(t+k) / \psi(t))+\phi$

$\Rightarrow \psi(t+k) / \psi(t)(1-\phi)=1+\phi$

$\Rightarrow \psi(t+k) / \psi(t)=\frac{1+\phi}{1-\phi}=\zeta$

$\Rightarrow|\psi(t+k) / \psi(t)| \leq 1$

$\Rightarrow|\zeta| \leq 1$

لكل قيم 0

ولهذه تكون طريقة Crank-Nicholson غير مشروطة لكي تكون مستقرة أي لا ترتبط بشروط. 


\section{$\underline{R E F E R E N C E S}$}

[1] Atoull, J.A and B.B. King (2000) "Stabilized Finite Element Methods and Feed back control for Burger Equation", American control conference, PP. 25-31.

[2] Balogh, A. and M. Krstic (1998) "Burger's equation with nonlinear boundary feedback: $\mathrm{H}^{1}$ stability”, well-posed ness and simulation, http://www-ames.ucsd.edu/research/krstic.

[3] Balogh, A.; D. S. Gilliam, and V. I. Shubov (2001) "Stationary solutions for a boundary controlled burger's equation", Math. and computer modeling, vol. 33, PP. 21-37.

[4] Estevez, P. G. (1994) "Non-classical symmetries and the singular manifold method: the burgers and the burgers-huxley equations", $\mathbf{J}$. Phys. A: Math. Gen., Vol. 27, PP. 2113-2127.

[5] Kakuda, K. and N. Tosaka (1990) "The generalized boundary element approach to burger's equation", International J. for Numerical Methods in Engineering, Vol. 29,PP. 245-261.

[6] Leonenko, N.N. and Meluikovn (2001) "Renarmahzation and Homogenization of the solutions of heat equation with linear Potential and related Burgers equation with random data", Theory probab \& Math. Statistics 1,PP.27-64.

[7] Mitchell A.R. and D.F. Griffiths (1980) The Finite Difference Method In Partial Differential Equation, John Wiley \& Sons. chichester .New York .515-353.QA37479.40626.

[8] Shan Thakumar M. (1989) Computer Based Numerical Analysis, Khanna Publishers, 2.B nath market,Neisaraic Delhi 110006 India.

[9] Wany X.Y.; Z.S. Zhus and Y.K. Lu (1990) "Solitary Wave Solution of The Generalized Burgers-Huxley Equation", Phys.A: Math. Gen. 23, PP.271- 274.

[10] Zhaug D.S.; G.W. Wei, ; D. J. Kouri and Q.K. Hoffman (1997) "Burger's Equation with High Reynolds Number", J. Phys. Fluid 9(6),PP. $1853-1855$. 